\title{
Correction to: A pathway map of AXL receptor-mediated signaling network
}

\author{
Shobha Dagamajalu ${ }^{1}$ D. A. B. Rex ${ }^{1} \cdot$ Akhina Palollathil $^{1} \cdot$ Rohan Shetty $^{2} \cdot$ Guruprasad Bhat $^{3} \cdot$ Lydia W. T. Cheung $^{4}$. \\ T. S. Keshava Prasad ${ }^{1}$
}

Published online: 29 September 2020

(C) The International CCN Society 2020

\section{Correction to: Journal of Cell Communication and Signaling https://doi.org/10.1007/s12079-020-00580-5}

The author would like to replace the PMID citation found in the first page of the article under the Introduction section (2nd column, line 6) and replace it with a bibliographic citation. Below are the necessary changes that should be made:

from: ...activity of AXL (Linger et al. 2008, O'Bryan et al. 1991). Phosphorylation of the residues in the N-terminal domain induced by GAS6 has also been shown (Pao-Chun et al. 2009). In particular, phosphorylated Tyr702 may stabilize the conformation of the activation loop of AXL, promoting AXL activity (PMID: 28,724,631). AXL is known to extensively ex...

to: ...activity of AXL (Linger et al. 2008, O'Bryan et al. 1991). Phosphorylation of the residues in the N-terminal domain induced by GAS6 has also been shown (Pao-Chun et al. 2009).

In particular, phosphorylated Tyr702 may stabilize the conformation of the activation loop of AXL, promoting AXL activity (Gajiwala et al., 2017). AXL is known to extensively ex...

Also given below is the complete bibliographic information of the additional Gajiwala et al., 2017 reference:

Gajiwala, K. S., Grodsky, N., Bolanos, B., Feng, J., Ferre, R., Timofeevski, S., Xu, M., Murray, B. W., Johnson, T. W. \& Stewart, A. 2017. The Axl kinase domain in complex with a macrocyclic inhibitor offers first structural insights into an active TAM receptor kinase. J Biol Chem, 292, 15705-15716.

Publisher's note Springer Nature remains neutral with regard to jurisdictional claims in published maps and institutional affiliations.

The online version of the original article can be found at https://doi.org/ 10.1007/s12079-020-00580-5

Shobha Dagamajalu

shobha_d@yenepoya.edu.in

T. S. Keshava Prasad

keshav@yenepoya.edu.in

D. A. B. Rex

rexprem@yenepoya.edu.in

Akhina Palollathil

akhinap@yenepoya.edu.in

Rohan Shetty

shettyrohan@rediffmail.com

Guruprasad Bhat

guru02doc@yahoo.co.in

Lydia W. T. Cheung

lydiacwt@hku.hk

1 Center for Systems Biology and Molecular Medicine, Yenepoya Research Centre, Yenepoya (Deemed to be University), Mangalore 575018, India

2 Department of Surgical Oncology, Yenepoya Medical College, Yenepoya (Deemed to be University), Mangalore 575018, India

3 Department of Medical Oncology, Yenepoya Medical College, Yenepoya (Deemed to be University), Mangalore 575018, India

4 School of Biomedical Sciences, Li Ka Shing Faculty of Medicine, The University of Hong Kong, Hong Kong, Hong Kong 